\title{
VARIABILIDAD INTERANUAL DE CLOROFILA EN EL GOLFO DE CALIFORNIA
}

\section{GULF OF CALIFORNIA INTERANUAL CHLOROPHYLL VARIABILITY}

\author{
T. Leticia Espinosa-Carreón ${ }^{1}$ y J. Eduardo Valdez-Holguín ${ }^{2}$
}

\begin{abstract}
Resumen
El Golfo de California es caracterizado como un mar semicerrado y una cuenca de evaporación. Se ha divido en varias zonas físicas y biogeográficas, características dadas por la dinámica de cada zona. El objetivo de este trabajo fue el de conocer la variabilidad interanual de la clorofila de Septiembre de 1997 a Diciembre de 2002 bajo condiciones El Niño y La Niña. Se obtuvieron del sensor SeaWiFS los compuestos semanales de clorofila con resolución de $9 \mathrm{~km}$ por píxel, para el periodo de estudio. Los resultados más sobresalientes de la distribución promedio de clorofila sugiere que el Golfo de California puede dividirse en tres zonas: oligotrófica (boca del golfo), mesotrófica (parte central) y eutrófica (región costera y parte norte del golfo, incluida la región de las Grandes Islas). La estacionalidad de la clorofila indica la predominancia de la señal anual. Es evidente la variabilidad interanual producida por los eventos El Niño 1997-1998 y por La Niña 1998-2001. El efecto de El Niño 1997-1998 es fuerte sobre la distribución de clorofila, el cambio hacia condiciones frías de La Niña durante 1998-1999 se refleja en anomalías negativas de biomasa fitoplanctónica, pero en positivas en los años 2000 y 2001.

Palabras clave: clorofila, variabilidad interanual, El Niño, La Niña, Golfo de California, imágenes de satélite, sensores remotos
\end{abstract}

\begin{abstract}
The Gulf of California is characterized as a semi-closed sea and an evaporation basin. It has been divided into various physical and bio-geographical zones, due to characteristic dynamics of each zone. The goal of this paper was to study the interannual chlorophyll variability from September 1999 to December 2002 during El Niño and La Niña conditions. Weekly composites for the study period with $9 \mathrm{~km}$ per pixel resolution were obtained from SeaWiFS imagery data. The spatial distribution of chlorophyll means suggest that the Gulf of California can be divided into three zones: oligotrophic (gulf mouth), mesotrophic (central area) and eutrophic (coastal region, north area and the Big Islands). The seasonal chlorophyll variability indicates a dominant annual signal. The interannual variability of chlorophyll in the Gulf of California is evident from El Niño 1997-1998 and La Nñia 1998-2001 events. The effect on chlorophyll distribution is strong during El Niño 1997-1998, but the change to cold conditions La Niña during 1998-1999 is appreciated in negative anomalies, but in positives in 2000 and 2001 years.
\end{abstract}

Keywords: chlorophyll, interanual variability, El Niño, La Niña, Gulf of California, imagery data, remote sensing

\section{Introducción}

Los ecosistemas pelágicos son clasificados de acuerdo al control ejercido por el ambiente físico, para su adecuado estudio son agrupados de acuerdo al tipo de forzamiento que los generan, o bien, de acuerdo a las escalas principales que ocupan en espacio y tiempo. La estacionalidad es uno de los modos básicos de variabilidad en muchos ecosistemas, y si están presentes, pueden ser detectados por los sensores remotos (Kahru \& Mitchell, 2001; Kahru et al., 2004), estimulando a su vez el desarrollo de nuevos instrumentos y tecnologías para facilitar las observaciones en diferentes escalas sinópticas de espacio y tiempo. Como resultado de esta nueva capacidad de usar sensores remotos para estudiar el océano, pueden realizarse determinaciones de la variabilidad a escala global de diferentes procesos físicos y biológicos. Los sensores remotos proporcionan información de la concentración de pigmentos fotosintéticos de aproximadamente $22 \%$ de la capa eufótica (Kirk, 1994).

La dinámica de la comunidad fitoplanctónica es determinada por el forzamiento físico que varía de región a región y entre estaciones climáticas. Los factores que la afectan son principalmente la biomasa autotrófica por si misma, la radiación solar, la temperatura, la concentración de nutrientes, los metales traza disponibles, la historia de vida y la estructura taxonómica del fitoplancton (Platt \& Sathyendranath, 1999). Dentro del contexto físico es necesario considerar los procesos horizontales y verticales, el viento como fuente de mezcla y transporte vertical de nutrientes, la advección horizontal y la radiación solar. En respuesta a estos 
factores las células fitoplanctónicas presentan adaptaciones fisiológicas bajo diferentes condiciones ambientales (Platt \& Sathyendranath, 1999).

El fenómeno El Niño Oscilación Austral (ENOS) se presenta entre tres y ocho años, con fuertes efectos en el Océano Pacífico Tropical (Chávez et al., 1999). El Niño se inicia por anomalías en el campo de vientos en el Océano Pacifico Ecuatorial Oriental. La termoclina se hunde y se eleva el nivel del mar en la zona costera (Huyer \& Smith, 1985). Al hundirse la termoclina la temperatura aumenta en la capa superficial, por lo que las surgencias durante ese periodo transportan hacia la superficie agua cálida y con baja concentración de nutrientes. Durante 1997-98 se registró en el Océano Pacífico uno de los eventos de El Niño más fuertes, caracterizado por aguas cálidas y con baja productividad primaria (Lynn et al., 1998). Posteriormente hubo una transición hacia condiciones de aguas frías con un incremento en la productividad, asociadas a un evento La Niña (Hayward et al., 1999). El Océano Pacífico tropical permaneció en condiciones de La Niña desde 1999 y hasta principios del 2002 (Schwing et al., 2002).

Además de ser considerado como cuenca de evaporación, el Golfo de California establece condiciones especiales de entrada y salida, mezcla y hundimiento de las masas de agua, con la consecuente formación de frentes y giros, mismos que varían de acuerdo con la profundidad y época climática (SotoMardones et al., 1999). Presenta una alta productividad primaria (Gilbert \& Allen, 1943; Alvarez-Borrego \& Lara-Lara, 1991) en contraste con otros mares semicerrados como el Mediterraneo y Mar Rojo (Lavín et al., 1995), debido a la alta disponibilidad de nutrientes en la zona eufótica originada por la circulación termohalina (Bray \& Robles, 1991), mezcla vertical (Alvarez-Borrego \& Lara-Lara, 1991) y surgencias costeras principalmente en invierno y primavera (Badan-Dangon et al., 1985; Alvarez-Borrego et al., 1978). Estas condiciones varían a lo largo del golfo y de estación climática. Es por lo tanto, una de la regiones más importantes de pesca en México (Zeitzschel, 1969), sus recursos han sido explotados durante décadas, y recientemente se le ha considerado a nivel mundial como sector marino de conservación y manejo sustentable (Lluch-Cota et al., 2007).

Con base en las diferentes condiciones oceanográficas, físicas y biológicas, ha sido dividido en varias regiones (Gilbert \& Allen, 1943; Santamaría-del-Ángel et al., 1994a; Gaxiola-Castro et al., 1995; Arias-Aréchiga, 1998; Hidalgo-González \& Alvarez-Borrego, 2001; Kahru et al., 2004; ValdezHolguín et al., 1999), aunque generalmente se divide en tres zonas: norte, centro y sur (Roden \& Groves, 1959). El objetivo de este trabajo fue el estudiar la variabilidad estacional e interanual de la biomasa fitoplanctónica (utilizando como estimador a la concentración de clorofila) en el Golfo de California desde Septiembre de 1997 a Diciembre de 2002, considerando al Golfo de California como una unidad.

\section{Área de estudio}

El Golfo de California (Figura 1) es una mar marginal que establece comunicación con el Océano Pacífico a través de una boca de $220 \mathrm{~km}$ de ancho, tiene una longitud aproximada de $1100 \mathrm{~km}$ (Roden \& Emilsson, 1979). Se localiza entre los $20^{\circ}$ y $32^{\circ}$ de latitud norte y los $105.5^{\circ}$ y $114.5^{\circ}$ de longitud oeste en el Pacifico Oriental, y se encuentra orientado en una dirección Noroeste (NO) - Sureste (SE). El Golfo de California está situado entre la Península de Baja California al Oeste y las costas de Sonora y Sinaloa al Este, al norte limita con la desembocadura del río Colorado y al sur con una línea imaginaria desde la península de California hasta Cabo Corrientes en Jalisco, México.

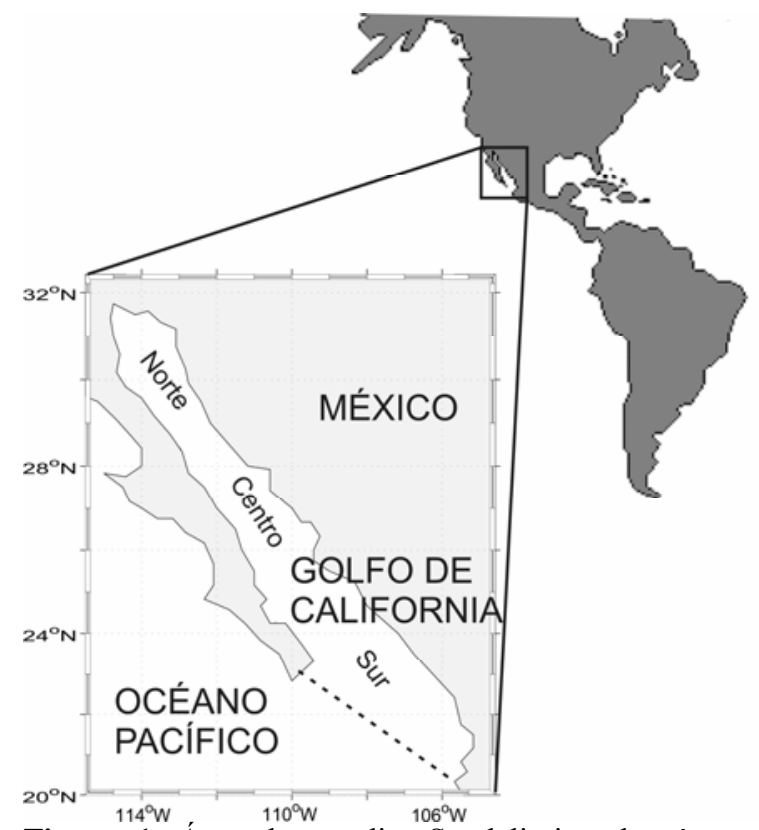

Figura 1. Área de estudio. Se delimitan las áreas Norte, Centro y Sur del Golfo de California.

\section{Metodología}

Se obtuvieron compuestos semanales globales de clorofila para el periodo de Septiembre de 1997 a Diciembre de 2002 del sensor Sea-viewing Wide Field of the view Sensor (SeaWiFS) del sitio ftp://oceans.gsfc.nasa.gov/SeaWiFS/Mapped/8Day/C

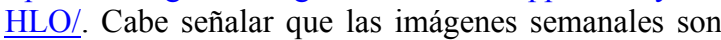
las que están a disposición de todo público (Nivel L3), las cuales han sido procesadas de acuerdo a los algoritmos bio-ópticos y atmosféricos correspondientes (Barnes et al. 1994). Estos compuestos tenían una resolución de $9 \mathrm{~km}$ por píxel y de estas imágenes globales se extrajo un área 
longitudinal ligeramente mayor a la comprendida para el Golfo de California, entre $20^{\circ} \mathrm{N}$ a $32^{\circ} \mathrm{N}$ y de $105^{\circ} \mathrm{W}$ a $115^{\circ} \mathrm{W}$. Santamaría-del-Ángel et al. (1994a) indican que el Golfo de California es un lugar frecuentemente libre de nubes, sin embargo, en este trabajo se decidió trabajar con imágenes limpias de nubes, por lo que se realizó una interpolación espacial para obtener las imágenes inicial y final libres de nubes y de datos anómalos. Posteriormente se realizó una interpolación espacial y temporal de todas las imágenes, obteniendo de esta manera imágenes sin nubes en todos los compuestos, la metodología de este procesamiento es la propuesta por Soto-Mardones (Comunicación personal) en donde a partir de la utilización del paquete computacional Mathematical Laboratory (Matlab, versión 6.5 () y con ayuda un programa de cómputo creado por el autor anteriormente mencionado, se aplican los comandos respectivos y se realiza la interpolación espacial y temporal, integrando los datos faltantes a partir de información de las mismas imágenes.

Se aplicó la siguiente ecuación para obtener los registros promedio por píxel para los 244 compuestos semanales, así como el ciclo estacional promedio de la serie de tiempo:

$$
F(x, t)=A_{0}(x)+A_{1}(x) \cos \left(w t-\varphi_{1}\right)+A_{2}(x) \cos \left(2 w t-\varphi_{2}\right)
$$

donde: $A_{0}, A_{1}, A_{2}$ corresponden a la amplitud temporal media, la amplitud anual y la amplitud semianual para cada serie de tiempo; $w=2 \pi / 365.25$ es la frecuencia anual; $\varphi_{1} \quad y \quad \varphi_{2}$ son las fases de los armónicos anual y semianual respectivamente, $t$ es el tiempo (año-día) y $\underline{x}$ es el número de datos de la serie de tiempo. Cabe señalar que los coeficientes de $A_{1}$ y $A_{2}$ no se presentan debido a que equivalen cada uno a una matriz de 18018 pixeles, obtenidos a partir de aplicar la ecuación 1 en toda la serie de tiempo (244 compuestos) y sólo se presenta el patrón promedio $\left(A_{0}\right)$ en la sección de resultados, así mismo, no se presentan los datos originales de las imágenes porque constituyen matrices de datos de $126 * 143 * 244$ pixeles.

Se realizó el cálculo del logaritmo natural a los datos y se estimó un ajuste polinomial para eliminar la tendencia y obtener sólo la influencia de la variabilidad estacional e interanual. Para realizar el cálculo de la anomalía estacional (señal anual más la señal semianual juntas), se eliminó el valor medio de las series de tiempo de clorofila, obteniéndose la curva representativa al ciclo estacional así como su porcentaje de explicación. Para obtener la anomalía no-estacional a las series de tiempo se les sustrajo el ciclo estacional (ecuación 1). Todo el procesamiento anterior se realizó con ayuda del paquete computacional Matlab (versión 6.5 $($ ), el módulo utilizado fue el de ajuste armónico.

Las series de tiempo mensuales de Enero de 1997 a Diciembre de 2002 de los índices climáticos
Multivariate ENSO Index (MEI) y el Pacific Decadal Oscilation (PDO) fueron obtenidos de los sitios: http://www.cdc.noaa.gov/Correlation/mei.data, http://jisao.washington.edu/pdo/img/v1v2PDOComp.p $\underline{\text { ng, respectivamente. A partir de la información de los }}$ índices MEI y PDO, y de la anomalía no-estacional de los datos, se realizó una prueba estadística de correlación cruzada, para obtener la co-variabilidad de los índices y datos a lo largo del tiempo.

\section{Resultados y discusión}

El patrón espacial promedio semanal de la biomasa fitoplanctónica observado de Septiembre de 1997 a Diciembre de 2002 (Figura 2) muestra un gradiente de concentración, desde una región oligotrófica de clorofila $\left(<0.2\right.$ miligramos por metro cúbico $\left.\left(\mathrm{mg} \cdot \mathrm{m}^{-3}\right)\right)$ en la región de la boca, incrementándose hacia una región mesotrófica $\left(0.2-1.0 \mathrm{mg} . \mathrm{m}^{-3}\right)$ en la parte central del golfo, y termina con una región eutrófica $(>1.0$ $\mathrm{mg} . \mathrm{m}^{-3}$ ) que comprende toda la región costera de Sinaloa, Sonora, Nayarit y parte de Jalisco, así como la región de las Grandes Islas (al norte del golfo). La región eutrófica costera llega a extenderse hasta más de $80 \mathrm{~km}$ hacia mar adentro.

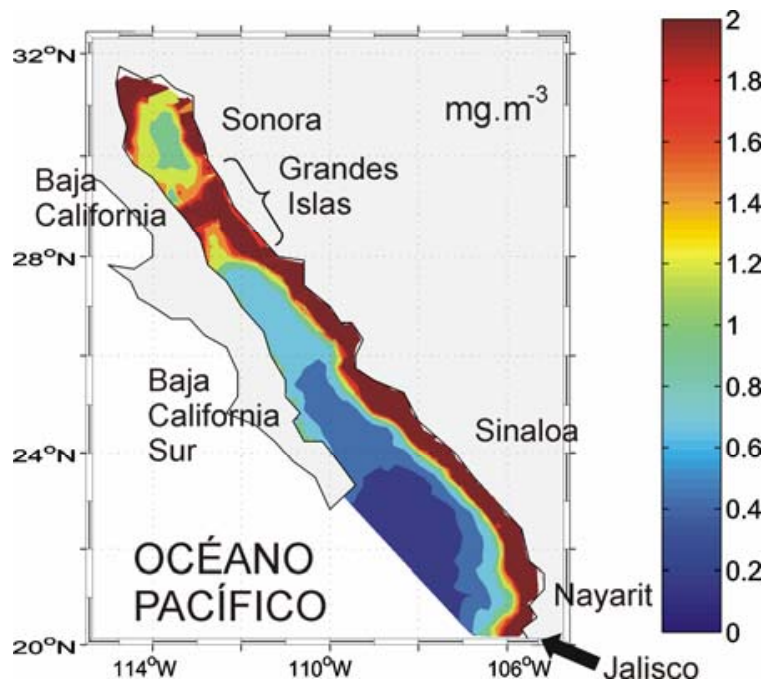

Figura 2. Concentración promedio de clorofila $\left(\mathrm{mg} . \mathrm{m}^{-3}\right)$ durante el período de estudio de Septiembre de 1997 a Diciembre de 2002.

El ciclo estacional de la biomasa fitoplanctónica explica el $51 \%$ de la varianza en el Golfo de California (Figura 3a, línea continua), con una predominancia anual (un valor máximo, entre Febrero y Marzo, y un mínimo entre Mayo y Junio, al año). En general, se observan anomalías con valores positivos durante invierno y primavera $\mathrm{y}$ negativos durante verano y otoño. El patrón promedio estacional está influenciado por las surgencias costeras de agua subsuperficial fría y rica en nutrientes, las cuales 
ocurren con mayor intensidad en invierno primavera sobre las costas de Sonora y Sinaloa (Roden, 1958; Alvarez-Borrego et al., 1978; Badán-Dagón et al., 1985), y promueven el incremento del fitoplancton a lo largo de la costa (Zeitzchel, 1969). La magnitud de estas surgencias está determinada por la intensidad de los vientos dominantes en las diferentes estaciones del año, la profundidad de la zona y las heterogeneidades de la línea de costa (Álvarez-Borrego \& Lara-Lara, 1991).
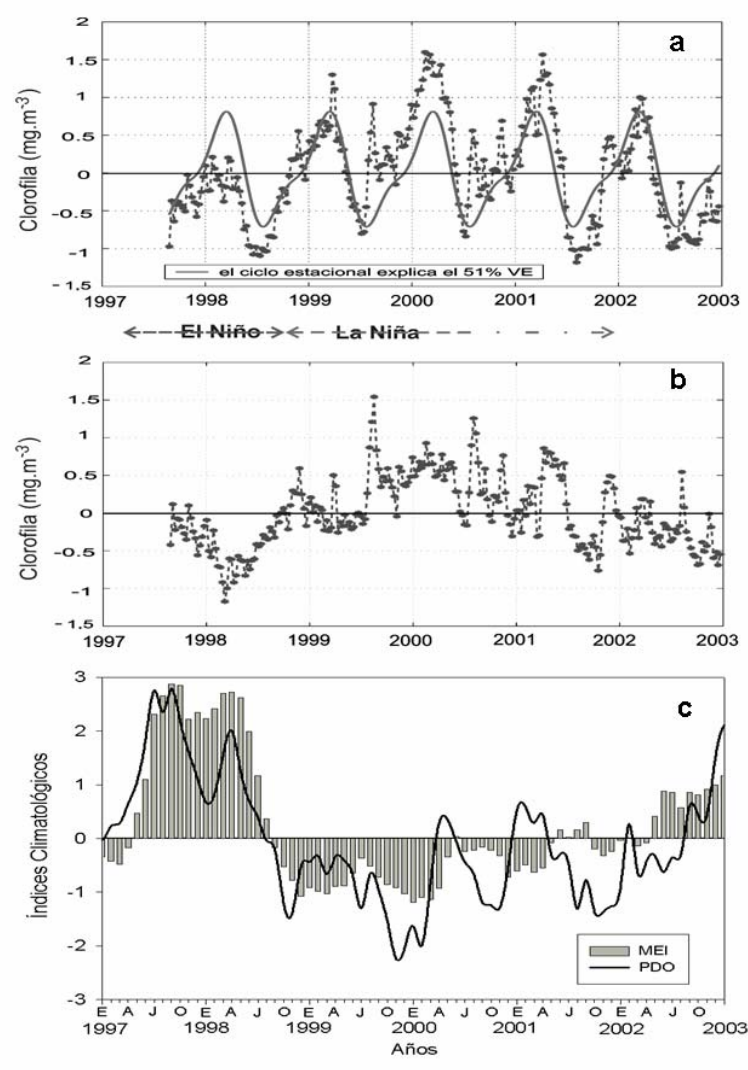

Figura 3. a) Anomalía de datos promedio de clorofila de Septiembre de 1997 a Diciembre de 2002 (asteriscos más línea punteada), ciclo estacional (línea continua), b) anomalía de datos promedio de clorofila de Septiembre de 1997 a Diciembre de 2002 con ciclo estacional removido. c) Índices Climáticos de Enero de 1997 a Diciembre de 2002, Multivariate ENOS Index (MEI) y Pacific Decadal Oscilation (PDO). en la parte inferior de la figura, E, A, J, O, indican los meses de Enero, Abril, Julio y Octubre respectivamente.

La pequeña inflexión que se observa en la curva del ciclo estacional en los meses de Agosto y Septiembre, sugiere que puede ser debida a que en alguna área específica del golfo podría dominar la señal semianual, tal como lo indica Kahru et al. (2004) para la región de las Grandes Islas. La amplitud estacional de cada variable física y ecológica se amplifica si consideramos al golfo como un todo (Lluch-Cota et al., 1999). La región de las Grandes Islas es caracterizada por una gran mezcla por mareas que produce movimiento vertical en la columna de agua hasta más de $500 \mathrm{~m}$ (Simpson et al., 1994). Los valores máximos de clorofila en esta región, a diferencia de la región central, ocurren durante verano (Valdez-Holguín et al., 1999), estos valores dominan la señal del ciclo estacional durante esta época.

En la serie de tiempo semanal de anomalías del promedio de los 244 compuestos de clorofila del SeaWiFS (Figura 3a, asteriscos + línea punteada) se aprecian algunas características muy importantes: 1) El Niño 1997-1998 hace que la clorofila disminuya y que se aprecien concentraciones de pigmentos de $0.5-$ $1.0 \mathrm{mg} . \mathrm{m}^{-3}$ menores a lo esperado por el ciclo estacional. 2) El efecto de La Niña 1999 es apenas apreciable y sólo en algunas semanas se registran valores de clorofila superiores a lo esperado. 3) Durante los años 2000, 2001 se presentan anomalías positivas superiores a lo esperado, cabe señalar que en entre Julio y Octubre del 2001 y a finales del 2002 se presentan de nuevo, anomalías negativas de clorofila.

Para realizar una identificación más apropiada de la influencia de los procesos interanuales de El Niño y La Niña en el Golfo de California, se restó el ciclo estacional a la serie de tiempo, obteniéndose una serie de tiempo no-estacional (sin señal estacional). En la Figura $3 \mathrm{~b}$ se muestra la variabilidad no-estacional donde se magnifica la influencia de los procesos interanuales en el Golfo de California. Durante 19971998 el Golfo de California reciente los efectos de El Niño, registrándose en los meses de SeptiembreDiciembre de 1997 valores de hasta 0.3 mg.m ${ }^{-3}$ menores a lo que se esperaba (cabe señalar que en la Figura $3 b$, los valores que se localizan cerca del cero son los que representan al ciclo estacional). A finales de Febrero y durante Marzo de 1998 se registran los valores más bajos, lo que indica que durante estos meses la concentración de clorofila fue hasta 1.0 mg. $\mathrm{m}^{-3}$ menor a lo esperado, esta disminución en clorofila coincide con los índices climáticos del Multivariate ENSO Index (MEI) y del Pacific Decadal Oscilation (PDO) que se presentan en la Figura 3c. Las anomalías negativas de clorofila coinciden con las anomalías positivas del MEI y del PDO. La covariabilidad de los índices MEI y el PDO contra la variabilidad no-estacional de los datos, indica un coeficiente significativo de $-0.56_{(\alpha=0.05 ; n=242)}$ y de -0.46 $(\alpha=0.05 ; \mathrm{n}=242)$ respectivamente. El MEI es un índice que permite monitorear al ENOA ya que integra mucha más información que refleja el acoplamiento del sistema océano-atmósfera, mientras que el PDO es derivado de las anomalías mensuales de temperatura 
superficial del mar en el Océano Pacífico Norte (Mantua et al., 1997).

Durante condiciones de El Niño, se registran altas temperaturas, altos niveles del mar, una termoclina más profunda, y la productividad primaria disminuye por una menor disponibilidad de nutrientes en la zona eufótica (Chávez et al., 2002). Hidalgo-González \& Alvarez-Borrego (2004) y Kahru et al. (2004) reportan para el norte del golfo, que los valores de producción primaria durante El Niño 1997-1998, fueron menores que en el resto del golfo. Valdez-Holguín \& Lara-Lara (1987) observaron que los efectos de El Niño 1983 no eran significativos en la región central, y explican que fenómenos físicos locales de enriquecimiento amortiguan los efectos de El Niño.

La región sur del golfo presenta la convergencia de tres diferentes masas de agua superficiales en la zona; el Agua del Golfo de California, el Agua Superficial Ecuatorial y el Agua Subsuperficial Subtropical, que determinan los procesos físicos, químicos y biológicos que se llevan a cabo en dicha región, aunado al gran forzamiento dinámico que ejerce el Océano Pacifico sobre la zona (Torres-Orozco, 1993). Esta dinámica ocasiona que la zona eufótica presente bajas concentraciones de nutrientes y oxígeno disuelto, con baja salinidad y alta temperatura, estas condiciones le confieren a la región sur del golfo sus características oligotróficas, con bajos valores de producción (Álvarez-Borrego \& Lara-Lara, 1991a). ValdezHolguín \& Lara-Lara (1987) concluyen que El Niño tiene mayores efectos en la región sur, con un decremento significativo en la clorofila. Santamaríadel-Ángel et al. (1994b) utilizando imágenes de satélite del sensor Coastal Zone Color Scanner (CZCS), reportan una disminución en la concentración de clorofila en la región sur del golfo durante El Niño 1982-1983. La producción primaria neta en la región sur del Golfo de California se encuentra asociada al evento El Niño y puede presentar consecuencias significativas en los niveles tróficos superiores (Kahru et al., 2004).

El mecanismo exacto de la reducción de la clorofila en el Golfo de California durante El Niño no se conoce con exactitud. Sin embargo, se puede mencionar que tanto la intrusión del Agua Superficial Tropical, la disminución de la mezcla vertical debido al incremento de la estratificación térmica (Kahru et al., 2004), y la dominancia de nanofitoplancton con bajo contenido de clorofila (Valdez-Holguín \& LaraLara, 1987) se conjugan para provocar dicha disminución.

Estudios previos de los efectos de El Niño en la Corriente de California indican una reducción en la intensidad de las surgencias costeras (Fiedler, 1984; Strub et al., 1990; Kahru \& Mitchell, 2000, 2002). Sin embargo, frente a Baja California se ha demostrado que se presenta un patrón positivo en la clorofila y en la producción primaria, ya que éstos se incrementan durante los eventos de El Niño 1982-1984 (Kahru \& Mitchell, 2000, 2002) y 1997-1998 (Kahru \& Mitchell, 2000, 2002; Espinosa-Carreón et al., 2004). Comparando los efectos de El Niño 1997-1998 en costas de Chile, Ulloa et al. (2001) reportan altos valores de temperatura y de oxígeno disuelto, sin embargo, ni la producción primaria ni la biomasa zooplanctónica se vieron disminuidos. Cabe señalar, que en el evento de El Niño 1982-1983, Thomas et al. (1994) indican que sólo se registran anomalías negativas de pigmentos en las costas del Perú y en zonas costeras que no exceden los $100 \mathrm{~km}$ de la costa, pero que dichas anomalías negativas no se registraron hacia latitudes de $20^{\circ} \mathrm{S}$.

Después de El Niño 1997-1998 se presenta un drástico cambio hacia condiciones La Niña, los índices climáticos (MEI y PDO) indican que las condiciones ambientales cambiaron de positivas en Agosto a negativas en Septiembre de 1998, prevaleciendo condiciones frías en general hasta finales de 2001 (Figura 3c). De acuerdo con Chávez et al. (2002) en condiciones de La Niña, se presentan bajas temperaturas, bajos niveles del mar, la termoclina es menos profunda, por lo que se incrementa la disponibilidad de nutrientes en la zona eufótica, y por lo tanto se presenta un incremento en la biomasa fitoplanctónica. Sin embargo, los resultados de este trabajo indican que no se aprecia la influencia de La Niña 1998-1999 en la clorofila en el Golfo de California. Este cambio climático no generó anomalías positivas de clorofila (Figura 3b), durante el periodo de Agosto de 1998 a Junio de 1999 sólo se registraron en algunos compuestos semanales anomalías positivas de clorofila. Incluso en los meses de Febrero y Marzo de 1999 se registraron anomalías negativas hasta de $\sim 0.3 \mathrm{mg} . \mathrm{m}^{-3}$, a pesar de que los índices climáticos (MEI y PDO) indican condiciones frías. En el Golfo de California continúa un lento cambio en respuesta a las condiciones ambientales, que podría estar acoplada a la oceanografía local de cada región. En un estudio realizado por Espinosa-Carreón et al. (2004) frente a Baja California, describen que los efectos de La Niña 1999 sobre la clorofila son poco apreciables y que la altas biomasas son producidas principalmente por efectos locales.

En el periodo de Septiembre de 1999 a Abril del 2001, el MEI describe tres pulsos negativos, y cambia a positivo a partir de Abril del 2002, con pequeñas oscilaciones entre Mayo de 2001 y Marzo de 2002. El PDO exhibe anomalías negativas desde Agosto de 1998 hasta Agosto de 2002, con pequeños cambios hacia anomalías positivas en los años 2000 y 2001 (Figura 3c). Lo anterior indica condiciones frías desde Agosto de 1998, pero que se reflejan en la concentración promedio de clorofila a partir de Septiembre de 1999 (Figura 3b). Dicho retrazo podría ser resultado de un "acondicionamiento" paulatino del golfo a las condiciones frías de La Niña, que de 
acuerdo al MEI comenzó desde Agosto de 1998, pero que en el Golfo de California fueron registrados hasta Septiembre de 1999 en las anomalías de clorofila. debido a que no se realizaron incubaciones de parámetros fotosintéticos que permitieran precisar con mayor detalle este supuesto.

Tabla 1. Datos promedio mensuales y su desviación estándar (entre paréntesis) de clorofila $\left(\mathrm{mg}^{-3} \mathrm{~m}^{-3}\right)$ de Septiembre de 1997 a Diciembre del 2002.

\begin{tabular}{|c|c|c|c|c|c|c|}
\hline Año & 1997 & 1998 & 1999 & 2000 & 2001 & 2202 \\
\hline Enero & & $0.84( \pm 0.85)$ & $1.30( \pm 2.17)$ & $2.09( \pm 2.63)$ & $1.28( \pm 2.00)$ & $1.30( \pm 1.69)$ \\
\hline Febrero & & $1.03( \pm 1.20)$ & $1.65( \pm 2.93)$ & $2.65( \pm 4.15)$ & $2.38( \pm 3.69)$ & $1.60( \pm 2.40)$ \\
\hline Marzo & & $0.77( \pm 0.85)$ & $1.57( \pm 2.37)$ & $3.13( \pm 6.21)$ & $2.95( \pm 2.42)$ & $2.59( \pm 4.17)$ \\
\hline Abril & & $0.87( \pm 1.01)$ & $1.44( \pm 1.97)$ & $3.08( \pm 5.35)$ & $2.98( \pm 4.53)$ & $2.17( \pm 3.59)$ \\
\hline Mayo & & $0.76( \pm 0.85)$ & $1.09( \pm 1.79)$ & $2.01( \pm 3.19)$ & $2.11( \pm 3.44)$ & $1.08( \pm 1.34)$ \\
\hline Junio & & $0.52( \pm 0.71)$ & $0.81( \pm 1.40)$ & $0.87( \pm 1.42)$ & $1.41( \pm 1.87)$ & $0.80( \pm 0.91)$ \\
\hline Julio & & $0.48( \pm 0.88)$ & $0.63( \pm 1.34)$ & $0.64( \pm 1.33)$ & $0.68( \pm 1.07)$ & $0.70( \pm 0.92)$ \\
\hline Agosto & & $0.50( \pm 0.95)$ & $1.59( \pm 3.82)$ & $1.54( \pm 2.35)$ & $0.58( \pm 1.01)$ & $0.90( \pm 1.63)$ \\
\hline Septiembre & $0.75( \pm 1.08)$ & $0.73( \pm 1.36)$ & $1.03( \pm 1.97)$ & $1.32( \pm 3.43)$ & $0.62( \pm 1.30)$ & $0.71( \pm 1.24)$ \\
\hline Octubre & $0.72( \pm 0.93)$ & $0.88( \pm 1.37)$ & $1.29( \pm 1.21)$ & $1.21( \pm 1.43)$ & $0.66( \pm 1.08)$ & $0.92( \pm 1.19)$ \\
\hline Noviembre & $0.87( \pm 1.07)$ & $1.18( \pm 1.60)$ & $1.60( \pm 2.44)$ & $1.91( \pm 2.95)$ & $1.60( \pm 2.55)$ & $1.14( \pm 1.44)$ \\
\hline Diciembre & $0.74( \pm 0.79)$ & $1.11( \pm 1.46)$ & $1.53( \pm 3.06)$ & $1.01( \pm 1.20)$ & $1.38( \pm 2.09)$ & $1.00( \pm 0.97)$ \\
\hline Promedio & 0.77 & 0.80 & 1.29 & 1.79 & 1.47 & 1.24 \\
\hline
\end{tabular}

Huntsman \& Barber (1977), indican que bajo condiciones de vientos fuertes, se genera una amplia capa de mezcla $(\sim 50 \mathrm{~m})$, y una limitada cantidad de luz para los procesos fotosintéticos. Rhyther et al. (1971) concluye que para las surgencias del Perú con vientos de $5 \mathrm{~m} . \mathrm{s}^{-1}$, se presentan condiciones óptimas para incrementar la productividad primaria. Thomas et al. (1994) reportan para la región del Perú, que entre el viento y los ciclos estacionales de pigmentos fotosintéticos, no se presenta una relación obvia. Sin embargo, Carr (2003) concluye que para condiciones simuladas de La Niña para las costas del Perú, la biomasa fitoplanctónica se ve reducida así como la fijación de carbono. Por su parte, Valdez-Holguín et al. (1999) y Gaxiola-Castro et al. (2002) indican para el Golfo de California, que debido a la fuerte mezcla vertical, se presenta una disminución en la capacidad fotosintética y por lo tanto, la productividad primaria se ve reducida. Lo anterior, podría sugerir que la biomasa fitoplanctónica registrada en condiciones $\mathrm{La}$ Niña 1998-1999, pudieran ser el producto de una disminución en la capacidad fotosintética del fitoplancton por los fuertes vientos presentes en el área, sin embargo, lo anterior no puede comprobarse
Kahru et al. (2004) reportan que las máximas concentraciones de producción primaria neta integrada en todo el Golfo de California se presentaron en los años 2000 y 2001, en Abril de 2001 se registraron valores de 23 teragramos de Carbono por mes (Tg.C.mes ${ }^{-1} \quad\left(\mathrm{Tg}=\right.$ teragramos $\left.=10^{15} \mathrm{~g}\right)$ ). La mínima concentración fue en Agosto de 1998 con 3.5 Tg.C.mes ${ }^{-1}$. En este trabajo los valores más altos de clorofila se registraron también en los años 2000 y 2001, en los meses de Febrero, Marzo y Abril, y los más bajos se registraron en los meses de Junio, Julio y Agosto de 1998, así mismo, el año con mayor cantidad de pigmentos fue el 2000 con un promedio de 1.79 mg.m ${ }^{-3}$, seguido del 2001 con 1.47 mg.m ${ }^{-3}$, los años donde se registraron las menores concentraciones fueron 1997 y 1998 con 0.77 mg.m ${ }^{-3}$ y 0.80 mg.m ${ }^{-3}$ respectivamente (Tabla1).

Schwing et al. (2002) reportan para el Sistema de la Corriente de California condiciones La Niña desde finales de 1998 hasta principios de 2002, condiciones que concuerdan en parte con la variabilidad interanual de clorofila en este trabajo. A partir de Abril de 2002 se aprecia un cambio de condiciones hacia otro evento El Niño (Figura 3c), aunque en la concentración de 
clorofila se registran anomalías negativas en este tiempo, no se registran anomalías negativas tan grandes como en El Niño 1997-1998 (Figura 3b), lo que sugiere que puede considerarse cómo un evento El Niño débil. McPhaden (2004) describe durante 20022003 un evento El Niño moderado para el Pacífico Ecuatorial. Durazo et al. (2005) describieron para la región frente a Baja California durante el periodo de 2002-2003 la presencia de un evento El Niño débil, donde no se observó una disminución de vientos dominantes o con dirección hacia el polo.

Con la finalidad de tener una mayor idea del patrón espacial de clorofila obtenida en los diferentes años, se escogieron compuestos representativos a cada evento. En la Figura 4a se muestra la concentración de clorofila durante El Niño 1997-1998 (Febrero-Marzo de 1998), se aprecia la baja concentración de clorofila en prácticamente todo el golfo, a excepción de una delgada franja en la zona zostera de Sonora y Sinaloa así como al norte. Sánchez-Velasco et al. (2001) reportaron un incremento de hasta 4 grados centígrados $\left({ }^{\circ} \mathrm{C}\right)$ en la temperatura del golfo durante 1997, con una disminución drástica de clorofila, para finales de 1998 los valores de temperatura y clorofila fueron normales. En condiciones de La Niña 19981999 (Figura 4b, Febrero-Marzo 1999), se registró un incremento en la concentración de clorofila principalmente en la zona costera y al norte del golfo. En Febrero-Marzo de 2000 (Figura 4c) se muestra el incremento de clorofila principalmente en tres áreas: norte del golfo, región de las Grandes Islas y frente a Sinaloa y al sur de ella, los valores registrados en estas áreas son superiores a los $2.0 \mathrm{mg} . \mathrm{m}^{-3}$. En FebreroMarzo de 2001 (Figura 4d) se observa una disminución de clorofila en las costas de Sinaloa, sin embargo, se incrementa en las áreas del norte y centro del golfo, la concentración de clorofila en dichas áreas también es superior a los $2.0 \mathrm{mg} . \mathrm{m}^{-3}$. Los resultados anteriores concuerdan con la producción primaria neta integrada en todo el golfo reportados por Kahru et al. (2004).

Con la finalidad de tener una mayor idea del patrón espacial de clorofila obtenida en los diferentes años, se escogieron compuestos representativos a cada evento. En la Figura 4a se muestra la concentración de clorofila durante El Niño 1997-1998 (Febrero-Marzo de 1998), se aprecia la baja concentración de clorofila en prácticamente todo el golfo, a excepción de una delgada franja en la zona zostera de Sonora y Sinaloa así como al norte. Sánchez-Velasco et al. (2001) reportaron un incremento de hasta 4 grados centígrados $\left({ }^{\circ} \mathrm{C}\right)$ en la temperatura del golfo durante 1997, con una disminución drástica de clorofila, para finales de 1998 los valores de temperatura y clorofila fueron normales. En condiciones de La Niña 19981999 (Figura 4b, Febrero-Marzo 1999), se registró un incremento en la concentración de clorofila principalmente en la zona costera y al norte del golfo.
En Febrero-Marzo de 2000 (Figura 4c) se muestra el incremento de clorofila principalmente en tres áreas: norte del golfo, región de las Grandes Islas y frente a Sinaloa y al sur de ella, los valores registrados en estas áreas son superiores a los $2.0 \mathrm{mg} \cdot \mathrm{m}^{-3}$. En FebreroMarzo de 2001 (Figura 4d) se observa una disminución de clorofila en las costas de Sinaloa, sin embargo, se incrementa en las áreas del norte y centro del golfo, la concentración de clorofila en dichas áreas también es superior a los $2.0 \mathrm{mg} . \mathrm{m}^{-3}$. Los resultados anteriores concuerdan con la producción primaria neta integrada en todo el golfo reportados por Kahru et al. (2004).

\section{Conclusiones}

La distribución promedio de clorofila indica que el Golfo de California puede dividirse en tres zonas: oligotrófica (boca del golfo), mesotrófica (parte central) y eutrófica (región costera y parte norte del golfo, incluida la región de las Grandes Islas). La estacionalidad de la clorofila indica la predominancia de la señal anual. Es evidente la variabilidad interanual de clorofila en el Golfo de California producida por los eventos El Niño 1997-1998 y por La Niña 1998-2001. El efecto de El Niño 1997-1998 es fuerte sobre la distribución de clorofila, presentándose principalmente en los meses de Febrero y Marzo de 1998, mostrando anomalías negativas hasta de 1.3 mg. $\mathrm{m}^{-3}$. El cambio hacia condiciones frías durante 1998 y 1999 no se refleja en un incremento de la concentración de clorofila en el golfo, sino al contrario, se presentan anomalías negativas hasta 0.3 $\mathrm{mg} \cdot \mathrm{m}^{-3}$. Lo anterior sugiere que en el Golfo de California, las condiciones frías de La Niña 19981999 se ven reflejadas en una disminución de la biomasa fitoplanctónica debida probablemente a una baja tasa fotosintética producida por la alta dinámica física del sistema.. Durante los años 2000 y 2001 continuaron las condiciones frías, apreciándose su efecto sobre la concentración de clorofila, registrándose las mayores anomalías positivas de clorofila en los meses de Febrero, Marzo y Abril en ambos años. En las condiciones de El Niño 2002-2003 se aprecian algunas anomalías negativas de clorofila.

Como comentario final, cabe señalar que se están realizando investigaciones sobre la regionalización de la clorofila en el Golfo de California (norte, centro y sur), para poder conocer así, con mayor detalle la variabilidad estacional e interanual de la clorofila en dichas zonas, asimismo, continuar con la producción primaria, que son de suma importancia para estimar el potencial pesquero que podría obtenerse en cierto tiempo y lugar. 

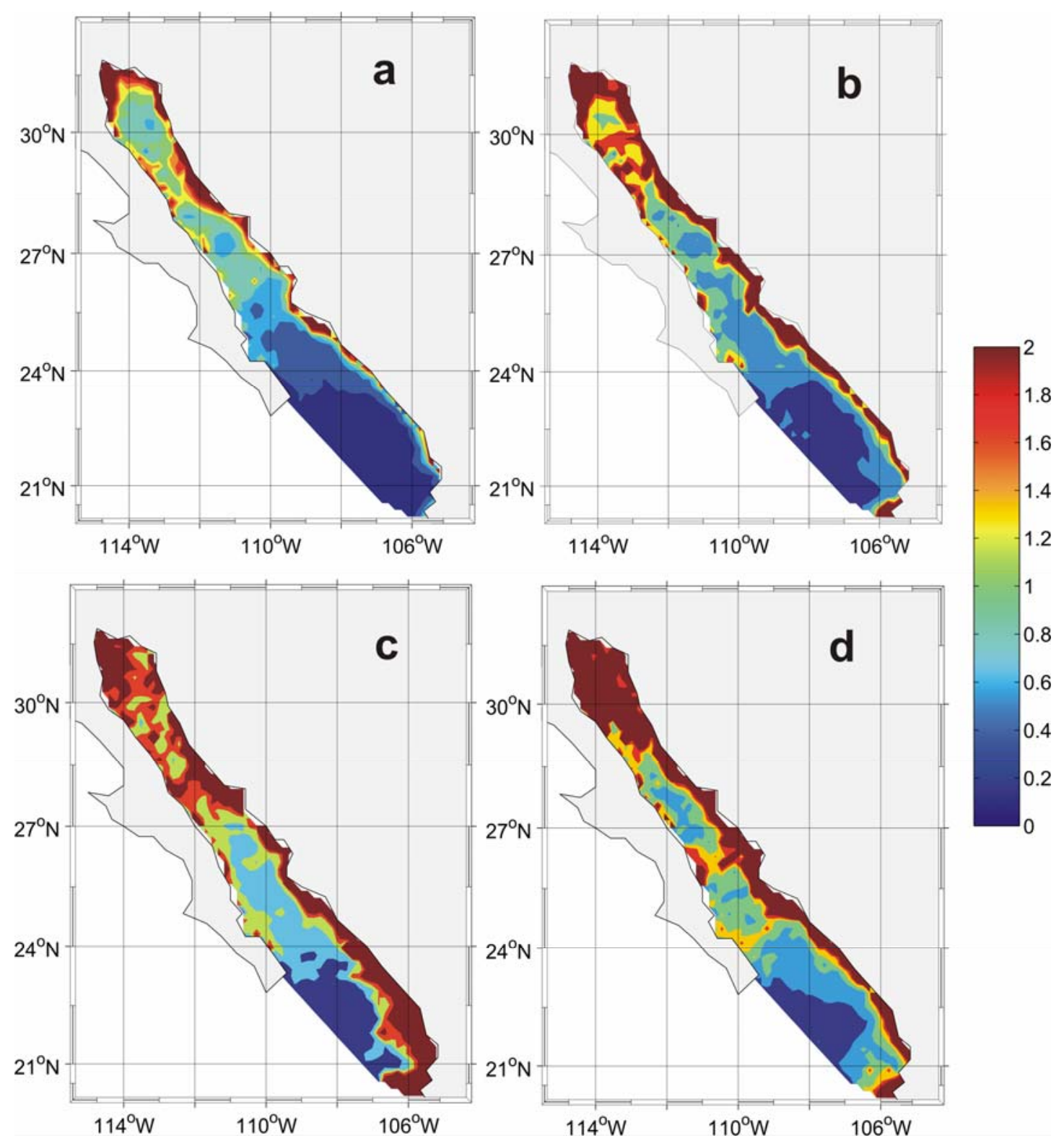

Figura 4. Distribución espacial de clorofila $\left(\mathrm{mg} \cdot \mathrm{m}^{-3}\right)$ : a) Febrero-Marzo de 1998 en condiciones El Niño, b) Febrero-Marzo de 1999, La Niña , c) Febrero-Marzo de 2000, La Niña , d) Febrero-Marzo de 2001, La Niña.

\section{Agradecimientos}

A los proyectos SIP-20060413 del Instituto Politécnico Nacional (IPN), CONACyT 23947, 45813, 60679 y SEP-2003-CO2-42569. El autor tiene becas EDI y COFAA del IPN. A la M. en C. Catalina Puente por proporcionar parte del material bibliográfico utilizado en este trabajo. Se agradecen los comentarios y sugerencias de los dos revisores anónimos, que sirvieron para elevar la calidad de este trabajo.

\section{Literatura citada}

Alvarez-Borrego S. \& Lara-Lara J.R. 1991. The physical enviroment and primary productivity of the Gulf of California. En: Dauphin JP \& Simoneit B (eds) The Gulf and Peninsular Province of the Californias. American Association of Petroleum Geologists.47, Tulsa: 555567.

Alvarez-Borrego S., Rivera J.A., Gaxiola-Castro G., AcostaRuíz M.J. \& Schwartzlose R.A. 1978. Nutrientes en el Golfo de California. Ciencias Marinas. 6: 13-22.

Arias-Aréchiga J.P. 1998. Regionalización del Golfo de California: una propuesta a partir de concentración de 
pigmentos fotosintéticos (CZCS). Tesis profesional. UABCS. México.

Badan-Dangon A., Koblinsky D.J. \& Baumgartner T. 1985. Spring and summer in the Gulf of California: observations of surface thermal patterns. Oceanologica Acta. 8: 13-22.

Barnes R.A., Holmes A.W., Barnes W.L., Esaias W.E., McClain C.R. \& Svitek T. 1994. "SeaWiFS Prelaunch Radiometric Calibration and Spectral Characterization". En: S.B. Hooker, E.R. Firestone y J.G. Acker (eds.). NASA Tech. Memo. 104566, vol. 23. NASA Goodard Space Flight Center, Greenbelt, Maryland.

Bray N.A. \& Robles J.M. 1991. Physical Oceanography of the Gulf of California. The Gulf and Peninsular Province ot the California. Memoir of the American association of Petroleum eologists.

Chávez F.P., Strutton P.G., Friederich G.E., Feely R.A., Foley G.C. \& McPhaden M.J. 1999. Biological and chemical response of the equatorial Pacific Ocean to the 1997-1998 El Niño". Science. 286: 2126-2131.

Pennington J.T., Castro C.G., Ryan J.P., Michisaki R.P., Schlining B., Walz P., Buck K.R., McPhaden A. \& Collins C.A.. 2002. Biological and chemical consequences of the 1997-1998 El Niño in central California waters. Progress in Oceanography. 54: 205-232.

Carr M.E. 2003. Simulation of carbon pathways in the plancktonic ecosystem off Peru during the 1007-1998 El Niño and La Niña. Journal of Geophysical Research, 108, No. C12, 3380.doi:10.1029/1999JC000064.

Durazo R., Gaxiola-Castro G., Lavaniegos B., CastroValdez R., Gómez-Valdés J. \& Mascarenhas Jr. A.D.S. 2005. Oceanographic conditions west of the Baja California coast, 2002-2003: A weak El Niño and subarctic water enhancement. Ciencias Marinas. 31: $537-552$.

Espinosa-Carreón T.L., Strub T., Beier E., Ocampo-Torres F. \& Gaxiola-Castro G. 2004. Seasonal and interannual variability of satellite-derived chlorophyll pigment, surface height, and temperature of Baja California. Journal Geophysical Reseacrh. 109, C03039. doi: 1029/2003JC002105.

Fiedler P.C. 1984. Satellite observations of the 1982-1983 El Niño along the U.S. Pacific coast. Science. 224: 1251-254.

Gaxiola-Castro G., Gracía-Córdova J., Valdez-Holguín J.E. \&. Botello-Ruvalcaba M. 1995. Spatial distribution of chlorophyll $a$ and primary productivity in relation to winter physical structure in the Gulf of California. Continental Shelf Research. 15: 1043-1059.

, Alvarez-Borrego S., Nájera-Martínez S., Zirino A.R. 2002. Internal waves effect on the Gulf of California phytoplankton. Ciencias Marinas. 28(3): 297309.

Gilbert J.Y. \& Allen W.E. 1943. The phytoplankton of the Gulf of California obtained by the E. W. Scripps in 1939 and 1940. Journal and Marine Research. 5: 89-110.

Hayward T.L., Baumgartner T.R., Checkley D.M., Durazo R., Gaxiola-Castro G., Hyrenbach K.D., Mantyla A.W., Mullin M.M., Murphree T., Schwing F.B., Smith P.E. \& Tegner M. 1999. The State of the California Current, 1998-1999: transition to cool-water conditions. California Cooperative Oceanic Fisheries Investigations Report. 40: 29-62.
Hidalgo-González R.M. \& Alvarez Borrego S. 2001. Chlorophyll profiles and the water column structure in the Gulf of California. Oceanológica Acta. 24: 19-28. . 2004.

Total and new production in the Gulf of California estimated from ocean color data from the satellite sensor SeaWiFS. Deep-Sea Research II. 51: 739-752.

Huntsman S.A. \& Barber R.T. 1977. Primary production off northwest Africa: the relationship to wind and nutrient condictions. Deep-Sea Research. 24: 25-33.

Huyer A. \& Smith R.L. 1985. The signature of El Niño off Oregon in 1982-83. Journal Geophysical Research. 90: 7133-7142.

Kahru M. \& Mitchell B.G. 2000. Influence of the 1997-98 El Niño on the surface chlorophyll in the California current. Geophysical Research Letters. 27: 2937-2940.

.2001. Seasonal and nonseasonal variability of satellite-derived chlorophyll and colored dissolved organic matter concentration in the California Current. Geophys. Res. Lett. 106: 2517-2529.

2002. Influence of the El Niño-

La Niña cycle on satellite-derived primary production in the California current. Geophysical Research Letters 29. doi:10.1029/2002GL014963.

, Marinone S.G., Lluch-Cota S.E., Parés-Sierra A., Mitchell G. 2004. Ocean color variability in the Gulf of California: scales from the El Niño-La Niña cycle to tides. Deep Sea Research II. 51: 139-146.

Kirk J.T.O. 1994. Light and photosynthesis in aquatic ecosystems. Cambridge University Press, New York.

Lavín M.F., Gaxiola-Castro G., Robles J.M. \& Richter. K. 1995. Winter water masses and nutrients in the northern Gulf of California. Journal of Geophysical Research. 100: 8587-8605.

Lara-Lara J.R. \& Valdez-Holguín J.E. 1988. Biomasa y productividad primaria del Golfo de California por fracción de tamaños durante la primavera de 1984. Ciencias Marinas. 14: 1-14.

Lluch-Cota S.E., Lluch-Cota D.B., Lluch-Belda D., Nevárez-Martínez M.O., Parés-Sierra A. \& HernándezVázquez S. 1999. Variability of sardine catch as related to enrichment, concentration, and retention processes in the central Gulf of California. California Cooperative Oceanic Fisheries Investigations Reports. 40: 184-190.

Lluch-Cota S.E., Aragón-Noriega E.A., Arreguín-Sánchez F., Aurioles-Gamboa D., Bautista-Romero J.J., Brusca R.C., Cervantes-Duarte R., Cortés-Altamirano R., DelMonte-Luna P., Esquivel-Herrera A., Fernández G., Hendrickx M.E., Hernández-Vázquez S., HerreraCervantes H., Kahru M., Lavín M., Lluch-Belda D., Lluch-Cota D.B., López-Martínez J., Marinote S.G., Nevárez-Martínez M.O., Ortega-García S., PalaciosCastro E., Parés-Sierra A., Ponce-Díaz G., RamírezRodríguez M., Salinas-Zavala C.A., Schwartzlose R.A. \& Sierra-Beltrán A.P. 2007. The Gulf of California: Review of ecosystem status and sustainability challenges. Progress in Oceanography. 73: 1-26.

Lynn R.J., Baumgartner T.R., García-Córdova J., Collins C., Hayward T., Hyrenbach D., Mantyla A., Murphree T., Shankle A., Schwing F., Sakuma K. \& Tegner M. 1998. The State of California Current, 1997-1998: Transition to El Niño conditions. California Cooperative Oceanic Fisheries Investigations Reports. 39: 25-49. 
Mantua N.J., Hare S.R., Zhang Y., Wallace J.M. \& Francis R.C. 1997. A Pacific interdecadal climate oscillation with impacts on salmon production. Bulletin of the American Meteorological Society. 78: 1069-1079.

McPhaden M.J. 2004. Evolution of the 2002-2003 El Niño. Bulletin of American Meteorogist Society. 85, 677, doi:10.1175/BAMS-85-5-677.

Platt T. \& Sathyendranath S. 1999. Spatial structure of pelagic ecosystem processes in the global ocean. Ecosystems. 2: 384-394.

Ryther J.H., Menzel D.W., Hulburt E.M., Lorenzen C.J. \& Corwin N. 1971. The production and utilization of organic matter in the Peru coastal current. Investigación Pesquera. 35(1): 43-59.

Roden G.I. 1958. Oceanographic and meteorological aspects of the Gulf of California. Pac. Sci. 12: 21-45.

Roden G.I. \& Emilson I. 1979. Physical oceanography of the Gulf of California. En: Ayala-Castañares A., F.B. Phleger, R. Schwartzlose \& A. Laguarda (Eds.) El Golfo de California: 1-46. Universidad Nacional Autónoma de México. México.

Roden G.I. \& Groves G.W. 1959. Recent Oceanographic Investigations in the Gulf of California. Journal Marine Research. 18: 10-35.

Sánchez-Velasco L., Valdez-Holguín J.E. Shirasago B., Cisneros-Mata M.A. \& Zarate A. 2001. Changes in the spawning environment of Sardinops caerulus in the Gulf of California during El Niño 1997-1998. Estuarine, Coastal and Shelf Science. 56: 1-11.

Santamaría-del-Ángel E., Álvarez Borrego S. \& MullerKarger F.E. 1994a. Gulf of California biogeographic regions based on Coastal Zone Color Scanner imagery. Journal of Geophysical Research. 99: 7411-7421.

. 1994b. The 1982-1984 El Niño in the Gulf of California as seen in Coastal Zone Color Scanner Imagery. Journal of Geophysical Research. 99: 7423-7431.

Schwing F.B., Bograd S.J., Collins C.A., Gaxiola-Castro G., García J., Goericke R.,. Gómez-Valdés J., Huyer A., Hyrenbach K.D., Kosro P.M., Lavaniegos B.E., Lynn R. J, Mantyla W., Ohman M.D., Peterson W.T., Smith R.L., Sydeman W.J., Venrick E. \& Wheeler P.A. 2002. The state of the California Current, 2001-2002: will the
California Current keep its cool, or is El Niño Looming?. California Cooperative Oceanic Fisheries Investigations Reports. 43: 31-73.

Simpson J.H., Souza A.J. \& Lavín M.F. 1994. Tidal mixing in the Gulf of California. En Breven KJ. Chatwin P.C. \& J.H. Milbank. (Eds.) Mixing and transport in the environment: 169-182. John Wiley and Sons. Ltd. London.

Soto-Mardones L., Marinone S.G., Parés-Sierra A. 1999. Variabilidad espacio temporal de la temperatura superficial del mar en el Golfo de California. Ciencias Marinas. 25: 1-30.

Strub P.T., James C., Thomas A. \& Abbot M. 1990 Seasonal and nonseasonal variability of satellite-derived surface pigments concentration in the California Current. Journal Geophysical Research. 95: 1150111530.

Torres-Orozco E. 1993. Análisis volumétrico de las masas de agua del Golfo de California. Tesis de Maestría. CICESE.

Thomas A.C., Hunag F., Strub P.T. \& James C. 1994. Comparison of the seasonal and interannual variability of phytoplankton pigment concentrations in the Peru and California Current systems. Journal of Geophysical Research, 99(C4): 7355-7370.

Ulloa O., Escribano R., Hormazabal S., Quiñones R.A., González R.R. \& Ramos M. 2001. Evolution and biological efects of the 1997-98 El Niño in the upwelling ecosystem northern Chile. Geophysical Research Letters. 28: 1591-1594.

Valdez-Holguín J.E. \& Lara-Lara R. 1987. Primary productivity of the Gulf of California of El Niño19821983 event. Ciencias Marinas. 13: 34-50.

, Alvarez-Borrego S. \& Trees C.C. 1999. Seasonal and spatial characterization of the Gulf of California phytoplankton photosynthesis parameters. Ciencias Marinas. 25: 445-467.

Zeitzschel B. 1969. Primary productivity in the Gulf of California. Marine Biolgy. 3: 201-207.

${ }^{1}$ Centro Interdisciplinario de Investigación para el Desarrollo Integral Regional (CIIDIR) Unidad Sinaloa - IPN. Blvd. Juan de Dios Bátiz Paredes \#250, Guasave, Sinaloa, México. C.P. 81101. Tel/Fax: (687) 8729625 y 8729626, tespinosac@ipn.mx; leticiaesp@,gmail.com

${ }^{2}$ Departamento de Investigación Científica de la Universidad de Sonora (DICTUS). Blvd.. Rosales y Rodríguez s/n, Col. Centro. Hermosillo, Sonora, México. C.P. 83000, Tel y Fax: (662) 259.2169 y (662) 259.2167. 